Delft University of Technology

\title{
On a comparison of Newton-Raphson solvers for power flow problems
}

\author{
Sereeter, Baljinnyam; Vuik, Cornelis; Witteveen, Cees
}

DOI

10.1016/j.cam.2019.04.007

Publication date

2019

Document Version

Final published version

Published in

Journal of Computational and Applied Mathematics

\section{Citation (APA)}

Sereeter, B., Vuik, C., \& Witteveen, C. (2019). On a comparison of Newton-Raphson solvers for power flow problems. Journal of Computational and Applied Mathematics, 360, 157-169.

https://doi.org/10.1016/j.cam.2019.04.007

\section{Important note}

To cite this publication, please use the final published version (if applicable).

Please check the document version above.

\section{Copyright}

Other than for strictly personal use, it is not permitted to download, forward or distribute the text or part of it, without the consent of the author(s) and/or copyright holder(s), unless the work is under an open content license such as Creative Commons.

\section{Takedown policy}

Please contact us and provide details if you believe this document breaches copyrights.

We will remove access to the work immediately and investigate your claim. 
Green Open Access added to TU Delft Institutional Repository 'You share, we take care!' - Taverne project

\section{https://www.openaccess.nl/en/you-share-we-take-care}

Otherwise as indicated in the copyright section: the publisher is the copyright holder of this work and the author uses the Dutch legislation to make this work public. 


\title{
On a comparison of Newton-Raphson solvers for power flow problems
}

\author{
Baljinnyam Sereeter*, Cornelis Vuik, Cees Witteveen \\ Faculty of Electrical Engineering, Mathematics and Computer Science, Delft University of Technology, Mekelweg 4, 2628 \\ $C D$, Delft, The Netherlands
}

\section{A R T I C L E I N F O}

\section{Article history:}

Received 5 April 2017

Received in revised form 14 December 2018

\section{Keywords:}

Power flow analysis

Newton-Raphson method

Power mismatch

Current mismatch

Polar

Cartesian

\begin{abstract}
A B S T R A C T
A general framework is given for applying the Newton-Raphson method to solve power flow problems, using power and current-mismatch functions in polar, Cartesian coordinates and complex form. These two mismatch functions and three coordinates, result in six possible ways to apply the Newton-Raphson method for the solution of power flow problems. We present a theoretical framework to analyze these variants for load (PQ) buses and generator (PV) buses. Furthermore, we compare newly developed versions in this paper with existing variants of the Newton power flow method. The convergence behavior of all methods is investigated by numerical experiments on transmission and distribution networks. We conclude that variants using the polar current-mismatch and Cartesian current-mismatch functions that are developed in this paper, performed the best result for both distribution and transmission networks.
\end{abstract}

(C) 2019 Elsevier B.V. All rights reserved.

\section{Introduction}

A power flow computation that determines the steady state behavior of the network is one of the most important tools for grid operators. The solution of a power flow computation can be used to assess whether the power system can function properly for the given generation and consumption. Therefore, power flow computations are performed in power system operation, control and planning.

The power flow or load flow problem is the problem of computing the voltage magnitude $\left|V_{i}\right|$ and angle $\delta_{i}$ in each bus of a power system where the power generation and consumption are specified. Over the years, various power flow solution techniques [1-15] have been developed on transmission networks. Gauss-Seidel (G-S), Newton power flow (N-R) and Fast Decoupled Load Flow (FDLF) based algorithms are the most widely used methods for the solution of transmission power flow problems. In practice, the Newton power flow method is preferred in terms of quadratic convergence and improved robustness [16]. Furthermore, many new methods [17-28] have been developed for distribution power flow problems and generally they are divided into two main categories such as modification of conventional power flow solution methods (GS, N-R, FDLF) and Backward-Forward Sweep (BFS)-based algorithms. Several reviews on distribution power flow solution methods can be found in [29-32].

In this paper, we focus on the Newton based power flow methods for balanced transmission and distribution networks. Depending on problem formulations (power or current mismatch) and coordinates (polar, Cartesian and complex form), the Newton-Raphson method can be applied in six different ways as a solution method for power flow problems. These six versions of the Newton power flow method are considered as the fundamental Newton power flow methods from which the further modified versions [8-15] are derived. Table 1 shows the previously published papers considering each variation of the Newton power flow method.

\footnotetext{
* Correspondence to: Department of Applied Mathematics, DIAM, Delft University of Technology, Mekelweg 4, 2628 CD, Delft, The Netherlands. E-mail addresses: b.sereeter@tudelft.nl (B. Sereeter), c.vuik@tudelft.nl (C. Vuik), c.witteveen@tudelft.nl (C. Witteveen).
} 
Table 1

Known versions of the Newton power flow method.

\begin{tabular}{llll}
\hline \multirow{2}{*}{ Mismatch formulation } & \multicolumn{3}{l}{ Coordinates } \\
\cline { 2 - 4 } & Polar & Cartesian & Complex form \\
\hline Power & {$[2]$} & {$[33]$} & {$[34]$} \\
Current & {$[35]$} & {$[35,36]$} & \\
\hline
\end{tabular}

The most widely used version is the Newton power flow method using the power-mismatch and polar coordinates which is introduced in [2]. In this method, the reactive power mismatch $\Delta Q$ and the voltage magnitude correction $\Delta V$ for each generator (PV) bus are eliminated from the Jacobian matrix equations and therefore the order of the equation is $\left(2 N-N_{g}-2\right)$.

In the version using the power-mismatch and Cartesian (rectangular) coordinates introduced in [33], the reactive power mismatch $\Delta Q$ is not eliminated from the Jacobian matrix equations for each PV bus but replaced by a voltage-magnitude-squared mismatch equation:

$$
\Delta|V|^{2}=\left(|V|^{s p}\right)^{2}-\left(V^{r}\right)^{2}+\left(V^{m}\right)^{2} .
$$

where $|V|^{s p}$ is specified voltage magnitudes and $V^{r}$ and $V^{m}$ are the real and imaginary parts of the complex voltages respectively. Therefore, the order of the Jacobian matrix equation is $(2 \mathrm{~N}-2)$ and it is concluded in [33] that the method is slightly less reliable and less rapid in convergence than the polar version developed in [2].

Although it is mentioned in $[2,16]$ that the complex power flow formulation does not mathematically lead to an analytic function of the complex voltage because of conjugate terms, the paper [34] investigated the version of the Newton power flow method using the power-mismatch in complex form. In paper [34], the Jacobian matrix equations are developed in complex form for each load (PQ) bus whereas two separate equations are created for each PV bus. The correction values of complex voltage for the PQ and PV buses are computed separately using different tolerances at each iteration. However, it is preferred to calculate correction values for both PQ and PV buses using common Jacobian matrix equations and the same tolerance.

The version using the current-mismatch and a mix of Cartesian and polar coordinates is discussed in [35]. In this method, each PQ bus is represented by two equations that are constructed from the real and imaginary parts of the complex current-mismatch function. A PV bus is represented by a single active power-mismatch $\Delta P$ and the voltagemagnitude-squared mismatch equation (1). The order of the Jacobian matrix equation is $(2 N-2)$ and it is concluded in [16] that these versions perform less satisfactorily than the power-mismatch versions.

The version using the current-mismatch and Cartesian coordinates is considered again in [36]. This method introduces a new dependent variable $\Delta Q$ for each PV bus and additional equations relating the corrections in polar and Cartesian coordinates:

$$
\begin{aligned}
\Delta|V| & =\frac{V^{r}}{|V|} \Delta V^{r}+\frac{V^{m}}{|V|} \Delta V^{m} \\
\Delta \delta & =\frac{V^{r}}{|V|^{2}} \Delta V^{m}-\frac{V^{m}}{|V|^{2}} \Delta V^{r} .
\end{aligned}
$$

Using Eqs. (2) and (3), this method makes the Jacobian matrix equation square in order to have a unique solution. In this method, the real $\Delta I^{r}$ and imaginary $\Delta I^{m}$ current-mismatch functions are expressed in terms of the real $\Delta P$ and reactive $\Delta Q$ power-mismatch functions. Then the reactive power-mismatch $\Delta Q$ is considered as a dependent variable for each PV bus and computed at each Newton iteration. Minor attempts were made to speed up the solution method using a partly constant approximation of the Jacobian during the iterations, but the results were not encouraging [36].

We did not find any discussion covering the Newton power flow method using the current-mismatch in complex form.

All variations of the Newton power flow method are developed by different researchers in different ways. This paper aims to discuss all six versions of the Newton power flow method using a common framework and to introduce new developments to improve the performance of other versions besides the most used version using the power-mismatch and polar coordinates [2]. Major improvements were done by us in Cartesian power-mismatch, polar current-mismatch and Cartesian current-mismatch versions. In versions using Cartesian coordinates, Eqs. (2) and (3) are used for PV buses instead of the voltage-magnitude-squared mismatch equation (1). In case of versions using the current-mismatch regardless of the choice of the coordinates, the reactive power $Q$ is considered as a dependent variable for each PV bus. Thus, we compute the correction $\Delta Q$ at each iteration and update $Q$ using the computed corrections. In case of the Cartesian power-mismatch, the order of the system is decreased to $\left(2 N-N_{g}-2\right)$ whereas [33] uses a system with the order $(2 N-2)$. The complex current-mismatch and complex power-mismatch versions are developed only for PQ buses.

This paper is structured as follows. In Section 2, a mathematical model of a power system is introduced briefly. Section 3 mathematically describes the power flow problem. The Newton-Raphson method and its all six versions for the solution of power flow problems are explained in Section 4. The numerical results of all solution techniques on balanced distribution and transmission networks, are presented in Section 5. Finally, the conclusion is given in Section 6. 
Table 2

Network bus type.

\begin{tabular}{llll}
\hline Bus type & Number of buses & Known & Unknown \\
\hline Slack node or swing bus & 1 & $\left|V_{i}\right|, \delta_{i}$ & $P_{i}, Q_{i}$ \\
Generator node or PV bus & $N_{g}$ & $P_{i},\left|V_{i}\right|$ & $Q_{i}, \delta_{i}$ \\
Load node or PQ bus & $N-N_{g}-1$ & $P_{i}, Q_{i}$ & $\left|V_{i}\right|, \delta_{i}$ \\
\hline
\end{tabular}

\section{Power system model}

Power systems are modeled as a network of buses and transmission lines whereas a network bus represents a system component such as a generator, load and transmission substation etc. There are three types of network buses such as a slack bus, a generator (PV) bus and a load (PQ) bus. Each bus in the power network is fully described by the following four electrical quantities:

$\left|V_{i}\right|$ : the voltage magnitude

$\delta_{i}$ : the voltage phase angle

$P_{i}$ : the active power

$Q_{i}$ : the reactive power

Depending on the type of the bus, two of the four electrical quantities are specified as shown in Table 2:

Here, $i$ is the index of the bus, $N_{g}$ is the number of generator buses and $N$ is the total number of buses in the network. For more details on the power system model we refer to [37].

\section{Power flow problem}

The power flow, or load flow, problem is the problem of computing the voltage magnitude $\left|V_{i}\right|$ and angle $\delta_{i}$ in each bus of a power system where the power generation and consumption are given. According to the Kirchhoff's Current Law $(\mathrm{KCL})$, the relation between the injected currents and bus voltages, is described by the admittance matrix $\mathbf{Y}$ :

$$
I=\mathbf{Y} V \leftrightarrow\left[\begin{array}{c}
I_{1} \\
I_{2} \\
\cdot \\
\cdot \\
I_{N}
\end{array}\right]=\left[\begin{array}{ccccc}
Y_{11} & Y_{12} & \cdot & \cdot & Y_{1 N} \\
Y_{21} & Y_{22} & \cdot & \cdot & Y_{2 N} \\
\cdot & \cdot & \cdot & \cdot & \cdot \\
\cdot & \cdot & \cdot & \cdot & \cdot \\
Y_{N 1} & Y_{N 2} & \cdot & \cdot & Y_{N N}
\end{array}\right]\left[\begin{array}{c}
V_{1} \\
V_{2} \\
\cdot \\
\cdot \\
V_{N}
\end{array}\right]
$$

where $I_{i}$ is the injected complex current, $V_{i}$ is the complex voltage at bus $i$ and $Y_{i j}$ is the element of the admittance matrix. From Eq. (4), the injected current at bus $i$ can be written as:

$$
I_{i}=\sum_{k=1}^{N} Y_{i k} V_{k} .
$$

The mathematical equations for the power flow problem are given by:

$$
\begin{aligned}
S_{i} & =V_{i} I_{i}^{*} \\
& =V_{i} \sum_{k=1}^{N} Y_{i k}^{*} V_{k}^{*}
\end{aligned}
$$

where $S_{i}$ is the injected complex power at bus $i$ and $I_{i}^{*}$ is the complex conjugate of the injected current. Mathematically, the power flow problem comes down to solving a nonlinear system of equations where all variables are given in complex numbers.

\section{Newton power flow solution methods}

The Newton based power flow methods use the Newton-Raphson (NR) method that is applied to solve a nonlinear system of equations $F(\vec{x})=0$. In NR method, the linearized problem is constructed as the Jacobian matrix equation

$$
-J(\vec{x}) \Delta \vec{x}=F(\vec{x})
$$

where $J(\vec{x})$ is the square Jacobian matrix and $\Delta \vec{x}$ is the correction vector. The Jacobian matrix is obtained by $J_{i k}=\frac{\partial F_{i}(\vec{x})}{\partial x_{k}}$ and is highly sparse in power flow applications [2,16]. The iteration process of the Newton based power flow method is shown in Algorithm 1. 


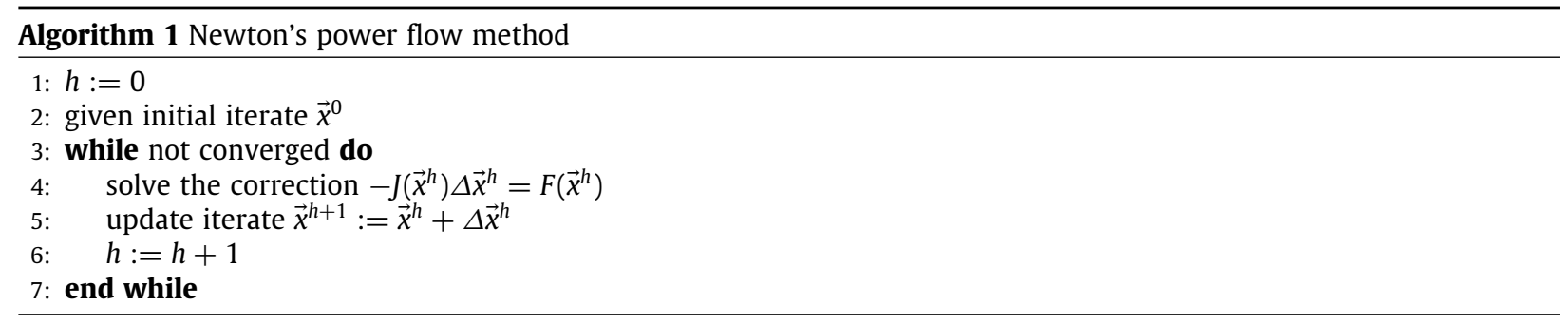

Table 3

Variable $\vec{x}$ in different coordinates.

\begin{tabular}{ll}
\hline Coordinates & Variable $\vec{x}$ \\
\hline Polar $\left(V_{i}=\left|V_{i}\right| e^{\mid \delta_{i}}\right)$ & {$\left[\delta_{1}, \ldots, \delta_{N},\left|V_{1}\right|, \ldots,\left|V_{N}\right|\right]^{T}$} \\
Cartesian $\left(V_{i}=V_{i}^{r}+\imath V_{i}^{m}\right)$ & {$\left[V_{1}^{m}, \ldots, V_{N}^{m}, V_{1}^{r}, \ldots, V_{N}^{r}\right]^{T}$} \\
Complex form $\left(V_{i}\right)$ & {$\left[V_{1}, \ldots, V_{N}\right]^{T}$} \\
\hline
\end{tabular}

Traditionally, direct solvers are used to solve the Jacobian matrix equation (8). Convergence of the method is mostly measured in the residual norm $\left\|F\left(\vec{x}^{h}\right)\right\|$ or relative residual norm $\left\|\frac{F\left(\vec{x}^{h}\right)}{F\left(\vec{x}^{0}\right)}\right\|$ of the mismatch function $F\left(\vec{x}^{h}\right)$ at each iteration. The Newton power flow method has a quadratic convergence when iterates are close enough to the solution. The Newton power flow methods formulate $F(\vec{x})$ as power or current mismatch functions and designate the unknown bus voltages and angles as the problem variables $\vec{x}$ using three different coordinates such as polar, Cartesian and complex form. Table 3 displays the problem variables defined in different coordinates.

\subsection{Power-mismatch formulation:}

The power flow problem (7) is formulated as the power-mismatch function $F(\vec{x})$ as follows:

$$
\begin{aligned}
F_{i}(\vec{x})=\Delta S_{i}(\vec{x}) & =S_{i}^{s p}-S_{i}(\vec{x}) \\
& =S_{i}^{s p}-V_{i} \sum_{k=1}^{N} Y_{i k}^{*} V_{k}^{*} \quad \forall i \in 1 \ldots N
\end{aligned}
$$

where $S_{i}^{s p}=P_{i}^{s p}+\imath Q_{i}^{s p}$ is the specified complex power injection at bus $i$. In general, specified active power $P_{i}^{s p}$ and reactive power $Q_{i}^{s p}$ injections at bus $i$ are given by following equations:

$$
\begin{aligned}
P_{i}^{s p} & =P_{i}^{G}-P_{i}^{L} \\
Q_{i}^{s p} & =Q_{i}^{G}-Q_{i}^{L}
\end{aligned}
$$

where $P_{i}^{G}$ and $Q_{i}^{G}$ are specified active and reactive power generation whereas $P_{i}^{L}$ and $Q_{i}^{L}$ are specified active and reactive power loads respectively. In this paper, $P_{i}^{L}$ and $Q_{i}^{L}$ are modeled as constant power loads.

The complex power-mismatch function (9) is separated into real equations and variables using polar and Cartesian coordinates. Table 4 shows power-mismatch functions in different coordinates. An application of the first order Taylor approximation to the power-mismatch functions results in a linear system of Eqs. (8) that is solved at each Newton iteration. Table 5 presents all equations computing the elements of the Jacobian matrix in different coordinates which are the partial derivatives of the power-mismatch function.

\subsubsection{Polar power-mismatch version (NR-p-pol [2])}

The Jacobian matrix equation (8) derived from the power-mismatch function in polar coordinates is given in the partitioned form for convenience of presentation:

$$
-\left[\begin{array}{l|l}
J^{11} & J^{12} \\
\hline J^{21} & J^{22}
\end{array}\right]\left[\begin{array}{c}
\Delta \delta \\
\Delta|V|
\end{array}\right]=\left[\begin{array}{c}
\Delta P \\
\Delta Q
\end{array}\right]
$$

where all sub-matrices are computed as $J^{11}=\frac{\partial \Delta P}{\partial \delta}, J^{12}=\frac{\partial \Delta P}{\partial|V|}, J^{21}=\frac{\partial \Delta Q}{\partial \delta}$ and $J^{22}=\frac{\partial \Delta Q}{\partial|V|}$. The Jacobian matrix equation (12) has to be modified for all PV buses since the voltage magnitude $\left|V_{j}\right|$ is specified instead of the reactive power $Q_{j}$ at each PV bus $j$. Therefore, $Q_{j}^{s p}$ cannot be computed and $\Delta Q_{j}$ cannot be formulated for each PV bus $j$. All partial derivatives of it with respect to voltage magnitude $\left|V_{i}\right|$ and angle $\delta_{i}$ cannot be taken. Similarly, $\Delta\left|V_{j}\right|$ does need to be computed for 
Table 4

Power-mismatch function in different coordinates.

\begin{tabular}{ll}
\hline Coordinates & Power-mismatch function: $F_{i}(\vec{x})=\Delta S_{i}(\vec{x})$ \\
\hline Polar & $\Delta P_{i}(\vec{x})=P_{i}^{s p}-\sum_{k=1}^{N}\left|V_{i}\right|\left|V_{k}\right|\left(G_{i k} \cos \delta_{i k}+B_{i k} \sin \delta_{i k}\right)$ \\
& $\Delta Q_{i}(\vec{x})=Q_{i}^{s p}-\sum_{k=1}^{N}\left|V_{i}\right|\left|V_{k}\right|\left(G_{i k} \sin \delta_{i k}-B_{i k} \cos \delta_{i k}\right)$ \\
\hline Cartesian & $\Delta P_{i}(\vec{x})=P_{i}^{s p}-\sum_{k=1}^{N}\left(V_{i}^{r}\left(G_{i k} V_{k}^{r}-B_{i k} V_{k}^{m}\right)+V_{i}^{m}\left(B_{i k} V_{k}^{r}+G_{i k} V_{k}^{m}\right)\right)$ \\
& $\Delta Q_{i}(\vec{x})=Q_{i}^{s p}-\sum_{k=1}^{N}\left(V_{i}^{m}\left(G_{i k} V_{k}^{r}-B_{i k} V_{k}^{m}\right)-V_{i}^{r}\left(B_{i k} V_{k}^{r}+G_{i k} V_{k}^{m}\right)\right)$ \\
\hline Complex form & $\Delta S_{i}(\vec{x})=S_{i}^{s p}-V_{i} \sum_{k=1}^{N} Y_{i k}^{*} V_{k}^{*}$ \\
\hline
\end{tabular}

Table 5

The partial derivatives of the power-mismatch function in different coordinates.

\begin{tabular}{|c|c|c|}
\hline Coordinates & $J_{i k}=\frac{\partial F_{i}(\vec{x})}{\partial x_{k}}$ & \\
\hline \multirow{8}{*}{ Polar } & \multirow{4}{*}{$i \neq k$} & $\frac{\partial \Delta P_{i}(\bar{x})}{\partial\left|V_{k}\right|}=-\left|V_{i}\right|\left(G_{i k} \cos \delta_{i k}+B_{i k} \sin \delta_{i k}\right)$ \\
\hline & & $\frac{\partial \Delta Q_{i}(\vec{x})}{\partial\left|V_{k}\right|}=-\left|V_{i}\right|\left(G_{i k} \sin \delta_{i k}-B_{i k} \cos \delta_{i k}\right)$ \\
\hline & & $\frac{\partial \Delta P_{i}(\vec{x})}{\partial \delta_{k}}=-\left|V_{i}\right|\left|V_{k}\right|\left(G_{i k} \sin \delta_{i k}-B_{i k} \cos \delta_{i k}\right)$ \\
\hline & & $\frac{\partial \Delta Q_{i}(\vec{x})}{\partial \delta_{k}}=-\left|V_{i}\right|\left|V_{k}\right|\left(-G_{i k} \cos \delta_{i k}-B_{i k} \sin \delta_{i k}\right)$ \\
\hline & \multirow{4}{*}{$i=k$} & $\frac{\partial \Delta P_{i}(\bar{x})}{\partial\left|V_{i}\right|}=-\left(2\left|V_{i}\right| G_{i i}+\sum_{i \neq k}\left|V_{k}\right|\left(G_{i k} \cos \delta_{i k}+B_{i k} \sin \delta_{i k}\right)\right)$ \\
\hline & & $\frac{\partial \Delta Q_{i}(\vec{x})}{\partial\left|V_{i}\right|}=-\left(-2\left|V_{i}\right| B_{i i}+\sum_{i \neq k}\left|V_{k}\right|\left(G_{i k} \sin \delta_{i k}-B_{i k} \cos \delta_{i k}\right)\right)$ \\
\hline & & $\frac{\partial \Delta P_{i}(\vec{x})}{\partial \delta_{i}}=-\sum_{i \neq k}\left|V_{i}\right|\left|V_{k}\right|\left(-G_{i k} \sin \delta_{i k}+B_{i k} \cos \delta_{i k}\right)$ \\
\hline & & $\frac{\partial \Delta Q_{i}(\vec{x})}{\partial \delta_{i}}=-\sum_{i \neq k}\left|V_{i}\right|\left|V_{k}\right|\left(G_{i k} \cos \delta_{i k}+B_{i k} \sin \delta_{i k}\right)$ \\
\hline \multirow[t]{6}{*}{ Cartesian } & \multirow[t]{2}{*}{$i \neq k$} & $\begin{array}{l}\frac{\partial \Delta P_{i}(\vec{x})}{\partial V_{k}^{r}}=-\left(V_{i}^{r} G_{i k}+V_{i}^{m} B_{i k}\right) \\
\frac{\partial \Delta Q_{i}(\vec{x})}{\partial V_{k}^{r}}=-\left(V_{i}^{m} G_{i k}-V_{i}^{r} B_{i k}\right) \\
\frac{\partial \Delta P_{i}(\vec{x})}{\partial V_{k}^{m}}=-\left(V_{i}^{m} G_{i k}-V_{i}^{r} B_{i k}\right)\end{array}$ \\
\hline & & $\frac{\partial \Delta Q_{i}(\vec{x})}{\partial V_{k}^{m}}=V_{i}^{r} G_{i k}+V_{i}^{m} B_{i k}$ \\
\hline & \multirow{4}{*}{$i=k$} & $\frac{\partial \Delta P_{i}(\vec{x})}{\partial V_{i}^{r}}=-\left(V_{i}^{r} G_{i i}+V_{i}^{m} B_{i i}+\sum_{k=1}^{N}\left(G_{i k} V_{k}^{r}-B_{i k} V_{k}^{m}\right)\right)$ \\
\hline & & $\frac{\partial \Delta Q_{i}(\vec{x})}{\partial V_{i}^{r}}=-\left(V_{i}^{m} G_{i i}-V_{i}^{r} B_{i i}+\sum_{k=1}^{N}\left(B_{i k} V_{k}^{r}+G_{i k} V_{k}^{m}\right)\right)$ \\
\hline & & $\frac{\partial \Delta P_{i}(\vec{x})}{\partial V_{i}^{m}}=-\left(V_{i}^{m} G_{i u}-V_{i}^{r} B_{i i}+\sum_{k=1}^{N}\left(B_{i k} V_{k}^{r}+G_{i k} V_{k}^{m}\right)\right)$ \\
\hline & & $\frac{\partial \Delta Q_{i}(\bar{x})}{\partial V_{i}^{m}}=V_{i}^{r} G_{i i}+V_{i}^{m} B_{i i}-\sum_{k=1}^{N}\left(G_{i k} V_{k}^{r}-B_{i k} V_{k}^{m}\right)$ \\
\hline \multirow[t]{2}{*}{ Complex form } & $i \neq k$ & $\frac{\partial \Delta S_{i}(\vec{x})}{\partial V_{k}}=-V_{i} Y_{i k}^{*}$ \\
\hline & $i=k$ & $\frac{\partial \Delta S_{i}(\vec{x})}{\partial V_{i}}=-\left(V_{i} Y_{i i}^{*}+I^{*}\right)$ \\
\hline
\end{tabular}

PV bus $j$ since $\left|V_{j}\right|$ is now known. Therefore, we eliminate all the $\frac{\partial \Delta P_{i}}{\partial\left|V_{j}\right|}, \frac{\partial \Delta Q_{i}}{\partial\left|V_{j}\right|}, \frac{\partial \Delta Q_{j}}{\partial \delta_{i}}$ and $\frac{\partial \Delta Q_{j}}{\partial\left|V_{i}\right|}$ from the Jacobian matrix $J(\vec{x})$, $\Delta\left|V_{j}\right|$ from the correction vector $\Delta \vec{x}$ and $\Delta Q_{j}$ from the power mismatch vector $F(\vec{x})$ for each PV bus $j$. The order of the resulting Jacobian matrix equation is $\left(2 N-N_{g}-2\right)$.

\subsubsection{Cartesian power-mismatch version (NR-p-car)}

The Jacobian matrix equation (8) is defined using the power-mismatch function in Cartesian coordinates as:

$$
-\left[\begin{array}{l|l}
J^{11} & J^{12} \\
\hline J^{21} & J^{22}
\end{array}\right]\left[\begin{array}{c}
\Delta V^{m} \\
\Delta V^{r}
\end{array}\right]=\left[\begin{array}{c}
\Delta P \\
\Delta Q
\end{array}\right]
$$

where all sub-matrices are given as $J^{11}=\frac{\partial \Delta P}{\partial V^{m}}, J^{12}=\frac{\partial \Delta P}{\partial V r}, J^{21}=\frac{\partial \Delta Q}{\partial V^{m}}$ and $J^{22}=\frac{\partial \Delta Q}{\partial V r}$. The Jacobian matrix equation (13) has to be modified for all PV buses for the same reason as we saw in 4.1.1. In this version, the reactive power-mismatch $\Delta Q_{j}$ cannot be formulated for each PV bus $j$ and therefore all partial derivatives $\frac{\partial \Delta Q_{j}}{\partial V_{k}^{m}}$ and $\frac{\partial \Delta Q_{j}}{\partial V_{k}^{r}}$ cannot be taken.

In paper [33], the reactive power mismatch $\Delta Q$ is replaced by a voltage-magnitude-squared mismatch equation (1) for all PV buses and therefore all partial derivatives $\frac{\partial \Delta Q_{j}}{\partial V_{k}^{m}}$ and $\frac{\partial \Delta Q_{j}}{\partial V_{k}^{r}}$ are also replaced by $\frac{\partial \Delta\left|V_{j}\right|^{2}}{\partial V_{k}^{m}}$ and $\frac{\partial \Delta\left|V_{j}\right|^{2}}{\partial V_{k}^{r}}$ respectively. 
Moreover, the order of the Jacobian matrix equation remains $(2 N-2)$ and it is concluded in [33] that the method is slightly less reliable and less rapid in convergence than the polar power-mismatch version 4.1.1.

In this paper, we develop a new approach that improves the performance of this version. In our approach, the reactive power-mismatch $\Delta Q_{j}$ is removed from the power-mismatch vector $F(\vec{x})$ for all PV buses and therefore all partial derivatives $\frac{\partial \Delta Q_{j}}{\partial V_{k}^{m}}$ and $\frac{\partial \Delta Q_{j}}{\partial V_{k}^{r}}$ are also eliminated from the Jacobian matrix $J(\vec{x})$. As a result of the elimination, the Jacobian matrix becomes a rectangular matrix. In order to make the Jacobian matrix square, we use Eq. (2) with $\Delta\left|V_{j}\right|=0$ since $\left|V_{j}\right|$ is now specified for each PV bus $j$. This gives us the relation between the corrections $\Delta V_{j}^{r}$ and $\Delta V_{j}^{m}$ as:

$$
\Delta V_{j}^{r}=-\frac{V_{j}^{m}}{V_{j}^{r}} \Delta V_{j}^{m}
$$

Using Eq. (14), the column of the Jacobian matrix with respect to the derivatives $\frac{\partial \Delta P_{i}}{\partial V_{j}^{r}}$ and $\frac{\partial \Delta Q_{i}}{\partial V_{j}^{r}}$ is added to the column with respect to the derivatives $\frac{\partial \Delta P_{i}}{\partial V_{j}^{m}}$ and $\frac{\partial \Delta Q_{i}}{\partial V_{j}^{m}}$ as follows:

$$
\begin{aligned}
& \frac{\partial \Delta P_{i}}{\partial V_{j}^{m}} \Delta V_{j}^{m}=\left(\frac{\partial \Delta P_{i}}{\partial V_{j}^{m}}-\frac{V_{j}^{m}}{V_{j}^{r}} \frac{\partial \Delta P_{i}}{\partial V_{j}^{r}}\right) \Delta V_{j}^{m} \\
& \frac{\partial \Delta Q_{i}}{\partial V_{j}^{m}} \Delta V_{j}^{m}=\left(\frac{\partial \Delta Q_{i}}{\partial V_{j}^{m}}-\frac{V_{j}^{m}}{V_{j}^{r}} \frac{\partial \Delta Q_{i}}{\partial V_{j}^{r}}\right) \Delta V_{j}^{m} .
\end{aligned}
$$

Now the correction $\Delta V_{j}^{r}$ can be eliminated from the correction vector $\Delta \vec{x}$ for each PV bus $j$ and therefore the order of the Jacobian matrix equation (13) is $\left(2 N-N_{g}-2\right)$.

\subsubsection{Complex power-mismatch version (NR-p-com)}

The Jacobian matrix equation (8) is computed using the power-mismatch function in complex form as:

$$
-[J][\Delta V]=[\Delta S]
$$

where the Jacobian matrix $J=\frac{\partial \Delta S}{\partial V}$ is obtained by taking the first order partial derivatives of the complex power-mismatch functions with respect to the complex voltage $V$. The Jacobian matrix equation (17) holds for all PQ buses but not for all PV buses because the complex power mismatch $\Delta S$ cannot be formulated for all PV buses. Therefore, this version can be applied to solve the power flow problem on networks with only a slack bus and PQ buses.

\subsection{Current-mismatch formulation:}

The current equation (5) and the power flow problem (6) are used to formulate the current-mismatch function $F(\vec{x})$ as follows:

$$
\begin{aligned}
F_{i}(\vec{x})=\Delta I_{i}(\vec{x}) & =I_{i}^{s p}-I_{i}(\vec{x}) \\
& =\left(\frac{S_{i}^{s p}}{V_{i}}\right)^{*}-\sum_{k=1}^{N} Y_{i k} V_{k} \quad \forall i \in 1 \ldots N
\end{aligned}
$$

where $I_{i}^{s p}=\left(\frac{S_{i}^{s p}}{V_{i}}\right)^{*}$ is the specified complex current injection at bus $i$.

The current-mismatch function (18) can be also expressed in terms of the power-mismatch function (9) as follows:

$$
\begin{aligned}
\Delta I_{i} & =\left(\frac{\Delta S_{i}}{V_{i}}\right)^{*} \quad \text { (complex) } \\
& =\frac{\cos \delta_{i} \Delta P_{i}+\sin \delta_{i} \Delta Q_{i}}{\left|V_{i}\right|}+l \frac{\sin \delta_{i} \Delta P_{i}-\cos \delta_{i} \Delta Q_{i}}{\left|V_{i}\right|} \quad \text { (polar) } \\
& =\frac{V_{i}^{r} \Delta P_{i}+V_{i}^{m} \Delta Q_{i}}{\left|V_{i}\right|^{2}}+l \frac{V_{i}^{m} \Delta P_{i}-V_{i}^{r} \Delta Q_{i}}{\left|V_{i}\right|^{2}} \quad \text { (Cartesian). }
\end{aligned}
$$

The complex current-mismatch function (18) is separated into real equations and variables using polar and Cartesian coordinates. Table 6 shows the current-mismatch functions in different coordinates. An application of a first order Taylor approximation to the current-mismatch function results in a linear system of Eqs. (8) that is solved in every Newton iteration. Table 7 gives all equations defining the elements of the Jacobian matrix in different coordinates.

\subsubsection{Polar current-mismatch version (NR-c-pol)}

The Jacobian matrix equation (8) is derived from the current-mismatch function in polar coordinates as:

$$
-\left[\begin{array}{l|l}
J^{11} & J^{12} \\
\hline J^{21} & J^{22}
\end{array}\right]\left[\begin{array}{c}
\Delta \delta \\
\Delta|V|
\end{array}\right]=\left[\begin{array}{c}
\Delta I^{r} \\
\Delta I^{m}
\end{array}\right]
$$


Table 6

Current-mismatch function in different coordinates.

\begin{tabular}{ll}
\hline Coordinates & Current-mismatch function: $F_{i}(\vec{x})=\Delta I_{i}(\vec{x})$ \\
\hline Polar & $\Delta I_{i}^{r}(\vec{x})=\frac{P_{i}^{s p} \cos \delta_{i}+Q_{i}^{s p} \sin \delta_{i}}{\left|V_{i}\right|}-\sum_{k=1}^{N}\left|V_{k}\right|\left(G_{i k} \cos \delta_{k}-B_{i k} \sin \delta_{k}\right)$ \\
& $\Delta I_{i}^{m}(\vec{x})=\frac{P_{i}^{s p} \sin \delta_{i}-Q_{i}^{s p} \cos \delta_{i}}{\left|V_{i}\right|}-\sum_{k=1}^{N}\left|V_{k}\right|\left(G_{i k} \sin \delta_{k}+B_{i k} \cos \delta_{k}\right)$ \\
\hline Cartesian & $\Delta I_{i}^{r}(\vec{x})=\frac{P_{i}^{s p} V_{i}^{r}+Q_{i}^{s p} V_{i}^{m}}{\left(V_{i}^{r}\right)^{2}+\left(V_{i}^{m}\right)^{2}}-\sum_{k=1}^{N}\left(G_{i k} V_{k}^{r}-B_{i k} V_{k}^{m}\right)$ \\
& $\Delta I_{i}^{m}(\vec{x})=\frac{P_{i}^{s p} V_{i}^{m}-Q_{i}^{s p} V_{i}^{r}}{\left(V_{i}^{r}\right)^{2}+\left(V_{i}^{m}\right)^{2}}-\sum_{k=1}^{N}\left(G_{i k} V_{k}^{m}+B_{i k} V_{k}^{r}\right)$ \\
\hline Complex form & $\Delta I_{i}(\vec{x})=\left(\frac{\left(s_{i}^{s p}\right.}{V_{i}}\right)^{*}-\sum_{k=1}^{N} Y_{i k} V_{k}$ \\
\hline
\end{tabular}

Table 7

The partial derivatives of the current-mismatch function in different coordinates.

\begin{tabular}{|c|c|c|}
\hline Coordinates & $J_{i k}=\frac{\partial F_{i}(\vec{x})}{\partial x_{k}}$ & \\
\hline \multirow{8}{*}{ Polar } & \multirow{4}{*}{$i \neq k$} & $\frac{\partial \Delta I_{i}^{r}(\vec{x})}{\partial\left|V_{k}\right|}=-\left(G_{i k} \cos \delta_{k}-B_{i k} \sin \delta_{k}\right)$ \\
\hline & & $\frac{\partial \Delta I_{i}^{m}(\vec{x})}{\partial\left|V_{k}\right|}=-\left(G_{i k} \sin \delta_{k}+B_{i k} \cos \delta_{k}\right)$ \\
\hline & & $\frac{\partial \Delta I_{i}^{r}(\vec{x})}{\partial \delta_{k}}=\left|V_{k}\right|\left(G_{i k} \sin \delta_{k}+B_{i k} \cos \delta_{k}\right)$ \\
\hline & & $\frac{\partial \Delta I_{i}^{m}(\vec{x})}{\partial \delta_{k}}=-\left|V_{k}\right|\left(G_{i k} \cos \delta_{k}-B_{i k} \sin \delta_{k}\right)$ \\
\hline & \multirow{4}{*}{$i=k$} & $\frac{\partial \Delta I_{i}^{r}(\vec{x})}{\partial\left|V_{i}\right|}=-\left(G_{i i} \cos \delta_{i}-B_{i i} \sin \delta_{i}\right)-\frac{P_{i}^{s p} \cos \delta_{i}+Q_{i}^{s p} \sin \delta_{i}}{\left|V_{i}\right|^{2}}$ \\
\hline & & $\frac{\partial \Delta I_{i}^{m}(\vec{x})}{\partial\left|V_{i}\right|}=-\left(G_{i i} \sin \delta_{i}+B_{i i} \cos \delta_{i}\right)-\frac{p_{i}^{s p} \sin \delta_{i}-Q_{i}^{s p} \cos \delta_{i}}{\left|V_{i}\right|^{2}}$ \\
\hline & & $\frac{\partial \Delta I_{i}^{r}(\vec{x})}{\partial \delta_{i}}=\left|V_{i}\right|\left(G_{i i} \sin \delta_{i}+B_{i i} \cos \delta_{i}\right)-\frac{P_{i}^{s p} \sin \delta_{i}-Q_{i}^{s p} \cos \delta_{i}}{\left|V_{i}\right|}$ \\
\hline & & $\frac{\partial \Delta I_{i}^{m}(\vec{x})}{\partial \delta_{i}}=-\left|V_{i}\right|\left(G_{i i} \cos \delta_{i}-B_{i i} \sin \delta_{i}\right)+\frac{P_{i}^{s p} \cos \delta_{i}+Q_{i}^{s p} \sin \delta_{i}}{\left|V_{i}\right|}$ \\
\hline \multirow{8}{*}{ Cartesian } & \multirow{4}{*}{$i \neq k$} & $\frac{\partial \Delta I_{i}^{r}(\vec{x})}{\partial V_{k}^{r}}=-G_{i k}$ \\
\hline & & $\frac{\partial \Delta I_{i}^{m}(\vec{x})}{\partial V_{k}^{r}}=B_{i k}$ \\
\hline & & $\frac{\partial \Delta I_{i}^{r}(\vec{x})}{\partial V_{k}^{m}}=B_{i k}$ \\
\hline & & $\frac{\partial \Delta I_{i}^{m}(\vec{x})}{\partial V_{k}^{m}}=-G_{i k}$ \\
\hline & \multirow{4}{*}{$i=k$} & $\frac{\partial \Delta I_{i}^{r}(\vec{x})}{\partial V_{i}^{r}}=-G_{i i}-\frac{P_{i}^{s p}\left(\left(V_{i}^{r}\right)^{2}-\left(V_{i}^{m}\right)^{2}\right)+2 V_{i}^{r} V_{i}^{m} Q_{i}^{s p}}{\left|V_{i}\right|^{4}}$ \\
\hline & & $\frac{\partial \Delta I_{i}^{m}(\vec{x})}{\partial V_{i}^{r}}=-B_{i i}+\frac{Q_{i}^{s p}\left(\left(V_{i}^{r}\right)^{2}-\left(V_{i}^{m}\right)^{2}\right)-2 V_{i}^{r} V_{i}^{m} P_{i}^{s p}}{\left|V_{i}\right|^{4}}$ \\
\hline & & $\frac{\partial \Delta l_{i}^{r}(\vec{x})}{\partial V_{i}^{m}}=B_{i i}+\frac{Q_{i}^{s p}\left(\left(V_{i}^{r}\right)^{2}-\left(V_{i}^{m}\right)^{2}\right)-2 V_{i}^{r} V_{i}^{m} P_{i}^{s p}}{\left|V_{i}\right|^{4}}$ \\
\hline & & $\frac{\partial \Delta I_{i}^{m}(\vec{x})}{\partial V_{i}^{m}}=-G_{i i}+\frac{P_{i}^{s p}\left(\left(V_{i}^{r}\right)^{2}-\left(V_{i}^{m}\right)^{2}\right)+2 V_{i}^{r} V_{i}^{m} Q_{i}^{s p}}{\left|V_{i}\right|^{4}}$ \\
\hline \multirow[t]{2}{*}{ Complex form } & $i \neq k$ & $\frac{\partial \Delta I_{i}(\vec{x})}{\partial V_{k}}=-Y_{i k}$ \\
\hline & $i=k$ & $\frac{\partial \Delta I_{i}(\vec{x})}{\partial V_{i}}=-\left(\frac{s_{i}^{s p}}{V_{i}^{2}}+Y_{i i}\right)$ \\
\hline
\end{tabular}

where all sub-matrices are computed as $J^{11}=\frac{\partial \Delta I^{r}}{\partial \delta}, J^{12}=\frac{\partial \Delta I^{r}}{\partial|V|}, J^{21}=\frac{\partial \Delta I^{m}}{\partial \delta}$ and $J^{22}=\frac{\partial \Delta I^{m}}{\partial|V|}$. Same as the polar powermismatch version 4.1.1, $\Delta\left|V_{j}\right|$ needs to be computed for each PV bus $j$ since $\left|V_{j}\right|$ is now known. Therefore, we eliminate all the $\frac{\partial \Delta I_{i}^{r}}{\partial\left|V_{j}\right|}$ and $\frac{\partial \Delta I_{i}^{m}}{\partial\left|V_{j}\right|}$ from the Jacobian matrix $J(\vec{x})$ and $\Delta\left|V_{j}\right|$ from the correction vector $\Delta \vec{x}$ for each PV bus $j$. As a result of the elimination, the Jacobian matrix becomes a rectangular matrix.

In paper [35], each PQ bus is represented by the real $\Delta I^{r}$ and imaginary $\Delta I^{m}$ current-mismatch functions. A PV bus is represented by the active power-mismatch $\Delta P$ and the voltage-magnitude-squared mismatch equation (1). Thus, the order of the Jacobian matrix equation is $(2 \mathrm{~N}-2)$ and it is concluded in [16] that these versions perform less satisfactorily than the power-mismatch versions. 
Table 8

The partial derivatives of the current-mismatch function in polar coordinates with respect to the reactive power $Q$.

\begin{tabular}{|c|c|c|}
\hline \multirow[t]{3}{*}{$J_{i j}=\frac{\partial F_{i}}{\partial x_{j}}$} & $i \neq j$ & $\begin{array}{l}\frac{\partial \Delta I_{i}^{r}(\vec{x})}{\partial Q_{j}}=0 \\
\frac{\partial \Delta I_{i}^{m}(\vec{x})}{\partial Q_{j}}=0\end{array}$ \\
\hline & $i=j$ & $\frac{\partial \Delta I_{j}^{r}(\vec{x})}{\partial Q_{j}}=\frac{\sin \delta_{j}}{\left|V_{j}\right|^{s p}}$ \\
\hline & & $\frac{\partial \Delta I_{j}^{r}(\vec{x})}{\partial Q_{j}}=-\frac{\cos \delta_{j}}{\left|V_{j}\right|^{s p}}$ \\
\hline
\end{tabular}

Table 9

The partial derivatives of the current-mismatch function in Cartesian coordinates with respect to the reactive power $Q$.

\begin{tabular}{ll}
\hline$J_{i j}=\frac{\partial F_{i}}{\partial x_{j}}$ & $\frac{\partial \Delta I_{i}^{r}(\vec{x})}{\partial Q_{j}}=0$ \\
\cline { 2 - 3 } & $\frac{\partial \Delta I_{i}^{m}(\vec{x})}{\partial Q_{j}}=0$ \\
& $\frac{\partial \Delta I_{j}^{r}(\vec{x})}{\partial Q_{j}}=\frac{V_{j}^{m}}{\left(V_{j}^{r}\right)^{2}+\left(V_{j}^{m}\right)^{2}}$ \\
& $\frac{\partial \Delta I_{j}^{r}(\vec{x})}{\partial Q_{j}}=\frac{-V_{j}^{r}}{\left(V_{j}^{r}\right)^{2}+\left(V_{j}^{m}\right)^{2}}$ \\
\hline
\end{tabular}

In our approach, the reactive power $Q_{j}$ is chosen as a dependent variable as $|V|$ and $\delta$ for each PV bus $j$ because we use the current-mismatch formulation directly. Since $Q_{j}$ is an unknown variable, all first order partial derivatives $\frac{\partial \Delta I_{i}^{r}}{\partial O_{j}}$ and $\frac{\partial \Delta I_{i}^{m}}{\partial Q_{j}}$ have to be computed as given in Table 8:

Now we add the derivatives $\frac{\partial \Delta I_{i}^{r}}{\partial Q_{j}}$ and $\frac{\partial \Delta I_{i}^{m}}{\partial Q_{j}}$ into the Jacobian matrix $J(\vec{x})$ and the correction $\Delta Q_{j}$ into the correction vector $\Delta \vec{x}$ for each PV bus $j$. As a result, the Jacobian matrix becomes a square again. The initial reactive power $Q_{j}^{0}$ at each PV bus $j$ is computed as follows:

$$
Q_{j}^{0}=\sum_{k=1}^{N}\left|V_{j}\right|\left|V_{k}\right|\left(G_{j k} \sin \delta_{j k}-B_{j k} \cos \delta_{j k}\right) .
$$

In each Newton iteration, the correction $\Delta Q_{j}$ is computed and the reactive power $Q_{j}$ is updated using the computed correction.

\subsubsection{Cartesian current-mismatch version (NR-c-car)}

The Jacobian matrix equation (8) is computed using the current-mismatch function in Cartesian coordinates as:

$$
-\left[\begin{array}{l|l}
J^{11} & J^{12} \\
\hline J^{21} & J^{22}
\end{array}\right]\left[\begin{array}{c}
\Delta V^{m} \\
\Delta V^{r}
\end{array}\right]=\left[\begin{array}{c}
\Delta I^{r} \\
\Delta I^{m}
\end{array}\right]
$$

where all sub-matrices are given as $J^{11}=\frac{\partial \Delta I^{r}}{\partial V^{m}}, J^{12}=\frac{\partial \Delta I^{r}}{\partial V^{r}}, J^{21}=\frac{\partial \Delta I^{m}}{\partial V^{m}}$ and $J^{22}=\frac{\partial \Delta I^{m}}{\partial V^{r}}$.

In paper [36], the real $\Delta I^{r}$ and imaginary $\Delta I^{m}$ current-mismatch functions are expressed in terms of the real $\Delta P$ and reactive $\Delta Q$ power-mismatch functions. Then the reactive power-mismatch $\Delta Q$ is considered as a dependent variable for each PV bus and computed at each Newton iteration. Minor attempts were made to speed up the solution method using a partly constant approximation of the Jacobian during the iterations, but the results were not encouraging [36].

In our approach, the reactive power $Q_{j}$ is chosen as a dependent variable for each PV bus $j$ as polar current-mismatch version 4.2.1. Since $Q_{j}$ is an unknown variable, all the first order partial derivatives $\frac{\partial \Delta I_{i}^{r}}{\partial Q_{j}}$ and $\frac{\partial \Delta I_{i}^{m}}{\partial Q_{j}}$ have to be computed as shown in Table 9:

Now we add the derivatives $\frac{\partial \Delta I_{i}^{r}}{\partial Q_{j}}$ and $\frac{\partial \Delta I_{i}^{m}}{\partial Q_{j}}$ into the Jacobian matrix $J(\vec{x})$ and the correction $\Delta Q_{j}$ into the correction vector $\Delta \vec{x}$ for each PV bus $j$. After the addition, the Jacobian matrix becomes a rectangular matrix. In order to make the Jacobian matrix square, we add the column of the Jacobian matrix with respect to the derivatives $\frac{\partial \Delta I_{i}^{r}}{\partial V_{j}^{r}}$ and $\frac{\partial \Delta I_{i}^{m}}{\partial V_{j}^{r}}$ to the column with respect to the derivatives $\frac{\partial \Delta I_{i}^{r}}{\partial V_{j}^{m}}$ and $\frac{\partial \Delta I_{i}^{m}}{\partial V_{j}^{m}}$ using (14) as follows:

$$
\frac{\partial \Delta I_{i}^{r}}{\partial V_{j}^{m}} \Delta V_{j}^{m}=\left(\frac{\partial \Delta I_{i}^{r}}{\partial V_{j}^{m}}-\frac{V_{j}^{m}}{V_{j}^{r}} \frac{\partial \Delta I_{i}^{r}}{\partial V_{j}^{r}}\right) \Delta V_{j}^{m}
$$


Table 10

Bus voltage corrections in different coordinates.

\begin{tabular}{|c|c|c|}
\hline Coordinates & Type of Bus & $\vec{x}^{h+1}:=\vec{x}^{h}+\Delta \vec{x}^{h}$ \\
\hline \multirow{3}{*}{ Polar } & \multirow{3}{*}{$P Q$ and PV } & $V_{i}^{(h+1)}=\left|V_{i}\right|^{(h+1)} e^{1 \delta_{i}^{(h+1)}}$ \\
\hline & & $\overline{|V|_{i}^{(h+1)}}=|V|_{i}^{(h)}+\Delta\left|V_{i}\right|^{(h)}$ \\
\hline & & $\delta_{i}^{(h+1)}=\delta_{i}^{(h)}+\Delta \delta_{i}^{(h)}$ \\
\hline \multirow{7}{*}{ Cartesian } & \multirow{3}{*}{$P Q$ and $P V$} & $V_{i}^{(h+1)}=\left(V_{i}^{r}\right)^{(h+1)}+l\left(V_{i}^{m}\right)^{(h+1)}$ \\
\hline & & $\overline{\left(V_{i}^{r}\right)^{(h+1)}=\left(V_{i}^{r}\right)^{(h)}+\left(\Delta V_{i}^{r}\right)^{(h)}}$ \\
\hline & & $\left(V_{i}^{m}\right)^{(h+1)}=\left(V_{i}^{m}\right)^{(h)}+\left(\Delta V_{i}^{m}\right)^{(h)}$ \\
\hline & \multirow{3}{*}{ PQ } & $V_{i}^{(h+1)}=\left|V_{i}\right|^{(h+1)} e^{l \delta_{i}^{(h+1)}}$ \\
\hline & & $\Delta\left|V_{j}\right|=\frac{V_{j}^{r}}{\left|V_{j}\right|} \Delta V_{j}^{r}+\frac{V_{j}^{m}}{\left|V_{j}\right|} \Delta V_{j}^{m}$ \\
\hline & & $\Delta \delta_{j}=\frac{V_{j}^{r}}{\left|V_{j}\right|^{2}} \Delta V_{j}^{m}-\frac{V_{j}^{m}}{\left|V_{j}\right|^{2}} \Delta V_{j}^{r}$ \\
\hline & PV & $\Delta \delta_{j}=\frac{\Delta V_{j}^{m}}{V_{j}^{r}}$ \\
\hline \multirow{2}{*}{ Complex } & $\mathrm{PQ}$ (NR-p-com) & $V_{i}^{(h+1)}=V_{i}^{(h)}+\left(\Delta V_{i}^{(h)}\right)^{*}$ \\
\hline & PQ (NR-c-com) & $V_{i}^{(h+1)}=V_{i}^{(h)}+\Delta V_{i}^{(h)}$ \\
\hline
\end{tabular}

$$
\frac{\partial \Delta I_{i}^{m}}{\partial V_{j}^{m}} \Delta V_{j}^{m}=\left(\frac{\partial \Delta I_{i}^{m}}{\partial V_{j}^{m}}-\frac{V_{j}^{m}}{V_{j}^{r}} \frac{\partial \Delta I_{i}^{m}}{\partial V_{j}^{r}}\right) \Delta V_{j}^{m} .
$$

Then the correction $\Delta V_{j}^{r}$ can be eliminated from the correction vector $\Delta \vec{x}$ for each PV bus $j$. The initial reactive power $Q_{j}^{0}$ at a PV bus $j$ is computed as follows:

$$
Q_{j}^{0}=\sum_{k=1}^{N}\left(V_{j}^{m}\left(G_{j k} V_{k}^{r}-B_{j k} V_{k}^{m}\right)-V_{j}^{r}\left(B_{j k} V_{k}^{r}+G_{j k} V_{k}^{m}\right)\right) .
$$

In each Newton iteration, the correction $\Delta Q_{j}$ is computed and the reactive power $Q_{j}$ is updated using the computed correction.

\subsubsection{Complex current-mismatch version (NR-c-com)}

The Jacobian matrix equation (8) is calculated using the current-mismatch function in complex form as:

$$
-[J][\Delta V]=[\Delta I]
$$

where the Jacobian matrix $J=\frac{\partial \Delta S}{\partial V}$ is obtained by taking the first order partial derivatives of the complex currentmismatch functions with respect to the complex voltage $V$. Same as the complex power-mismatch version 4.1.3, this version is applicable for the power flow problem on networks with only a slack bus and PQ buses.

Bus voltage corrections in different coordinates are given in Table 10:

\section{Numerical experiment}

The newly developed/improved versions of the Newton power flow method (Cartesian power-mismatch, polar current-mismatch, Cartesian current-mismatch and complex current-mismatch) discussed in Section 4, are compared to the existing versions of the Newton power flow method (polar power-mismatch [2], Cartesian power-mismatch [33] and Cartesian current-mismatch [36]) for the numerical experiments. Two distribution networks (DCase33 [38] and DCase69 [39]) and four transmission networks taken from Matpower [40] (case1354pegase, case2737sop, case9241pegase and case13659pegase) are used to test the convergence ability and scalability of all variants of the Newton power flow solution method. All methods are implemented in Matlab and the constant power load model is used for loads. The relative convergence tolerance is set to $10^{-5}$ and the maximum number of iterations is set to 10 . All experiments are performed on an Intel computer with four cores i5-4690 3.5 GHz CPU and 64Gb memory, running a Debian 64-bit Linux 8.7 distribution.

\subsection{Distribution networks}

The convergence result of all Newton power solution methods for two distribution network (DCase33 and DCase69) is shown in Table 11. 
Table 11

Distribution networks: DCase33 and DCase69.

\begin{tabular}{|c|c|c|c|c|c|c|}
\hline \multirow{3}{*}{ Methods } & \multicolumn{6}{|c|}{ Test cases } \\
\hline & \multicolumn{3}{|c|}{ DCase33 } & \multicolumn{3}{|c|}{ DCase69 } \\
\hline & iter & time & $\|F(\vec{x})\|_{\infty}$ & iter & time & $\|F(\vec{x})\|_{\infty}$ \\
\hline NR-p-pol [2] & 3 & 0.0072 & $7.4675 e-06$ & 4 & 0.0113 & $5.5875 \mathrm{e}-09$ \\
\hline NR-p-car & 3 & 0.0063 & $1.0433 \mathrm{e}-06$ & 3 & 0.0068 & $8.1777 e-06$ \\
\hline NR-p-car [33] & 3 & 0.0072 & $1.0897 e-06$ & 3 & 0.0081 & $8.0940 \mathrm{e}-06$ \\
\hline NR-p-com & 6 & 0.0055 & $6.4610 \mathrm{e}-06$ & 7 & 0.0063 & $4.0138 \mathrm{e}-06$ \\
\hline NR-c-pol & 3 & 0.0081 & $1.4291 \mathrm{e}-09$ & 3 & 0.0094 & $8.5226 \mathrm{e}-09$ \\
\hline NR-c-car & 3 & 0.0068 & $1.3954 \mathrm{e}-09$ & 3 & 0.0081 & $1.9503 \mathrm{e}-08$ \\
\hline NR-c-car [36] & 3 & 0.0111 & $1.3964 \mathrm{e}-09$ & 3 & 0.0124 & $1.9476 \mathrm{e}-08$ \\
\hline NR-c-com & 7 & 0.0055 & $5.3792 \mathrm{e}-06$ & 10 & 0.0076 & $2.7697 e-06$ \\
\hline
\end{tabular}

Table 12

Small transmission networks: TCase1354 and TCase2737.

\begin{tabular}{|c|c|c|c|c|c|c|}
\hline \multirow{3}{*}{ Methods } & \multicolumn{6}{|c|}{ Test cases } \\
\hline & \multicolumn{3}{|c|}{ TCase 1354} & \multicolumn{3}{|c|}{ TCase2737 } \\
\hline & iter & time & $\|F(\vec{x})\|_{\infty}$ & iter & time & $\|F(\vec{x})\|_{\infty}$ \\
\hline NR-p-pol [2] & 3 & 0.0284 & $6.2678 \mathrm{e}-06$ & 4 & 0.0640 & $1.5353 \mathrm{e}-08$ \\
\hline NR-p-car & 3 & 0.0265 & $1.5795 \mathrm{e}-06$ & 4 & 0.0634 & $2.3500 \mathrm{e}-06$ \\
\hline NR-p-car [33] & 3 & 0.0298 & $2.2486 \mathrm{e}-06$ & 5 & 0.0777 & $2.8518 \mathrm{e}-06$ \\
\hline NR-c-pol & 3 & 0.0313 & $8.3005 e-10$ & 4 & 0.0700 & $6.1735 \mathrm{e}-07$ \\
\hline NR-c-car & 3 & 0.0306 & $6.1446 \mathrm{e}-10$ & 4 & 0.0649 & $8.6780 \mathrm{e}-07$ \\
\hline NR-c-car [36] & 5 & 0.0507 & $9.9969 \mathrm{e}-06$ & 5 & 0.0838 & $7.9842 \mathrm{e}-07$ \\
\hline
\end{tabular}

Table 13

Large transmission networks: TCase9241 and TCase13659.

\begin{tabular}{|c|c|c|c|c|c|c|}
\hline \multirow{3}{*}{ Methods } & \multicolumn{6}{|c|}{ Test cases } \\
\hline & \multicolumn{3}{|c|}{ TCase9241 } & \multicolumn{3}{|c|}{ TCase13659 } \\
\hline & iter & time & $\|F(\vec{x})\|_{\infty}$ & iter & time & $\|F(\vec{x})\|_{\infty}$ \\
\hline NR-p-pol [2] & 6 & 0.3555 & $2.1292 \mathrm{e}-09$ & 5 & 0.3899 & $2.2891 \mathrm{e}-09$ \\
\hline NR-p-car & 5 & 0.2908 & $2.1026 \mathrm{e}-08$ & 6 & 0.4689 & $7.9833 \mathrm{e}-12$ \\
\hline NR-p-car [33] & 5 & 0.3180 & $2.0742 \mathrm{e}-06$ & 10 & 0.8899 & $1.401 \mathrm{e}+148$ \\
\hline NR-c-pol & 3 & 0.1973 & $6.4746 e-07$ & 4 & 0.3634 & $3.4366 \mathrm{e}-09$ \\
\hline NR-c-car & 3 & 0.1993 & $1.9438 \mathrm{e}-06$ & 4 & 0.3619 & $8.6170 \mathrm{e}-09$ \\
\hline NR-c-car [36] & 10 & 0.6595 & 0.0023 & 10 & 0.9036 & 1.1482 \\
\hline
\end{tabular}

We observe that variants using complex form (NR-p-com and NR-c-com) need more iterations and have a linear convergence compared to other variants. Thus, NR-p-com and NR-c-com are the least preferable variants of the Newton power flow method. Furthermore, remaining versions employing Polar and Cartesian coordinates converge after the same number of iterations. However, we discover that the residual norm of current-mismatch functions is much smaller than the residual norm of power-mismatch functions regardless of the choice of the coordinates. Thus, we can conclude that versions using current-mismatch functions are more suitable for solving distribution power flow problems than versions using power-mismatch functions. Variants polar current-mismatch (NR-c-pol) and Cartesian current-mismatch (NR-c-car) developed in this paper perform the best for two distribution networks in terms of both a number of iterations and the residual norm.

\subsection{Transmission networks}

Since the complex power-mismatch and complex current-mismatch versions are developed only for PQ buses, these variants are not applied to transmission power flow problems including PV buses. Tables 12 and 13 show the convergence result of all solution methods for transmission networks (TCase1354, TCase2737, TCase9241 and TCase13659).

For smaller transmission networks TCase 1354 and TCase2737, all versions result in the same behavior except the Cartesian current-mismatch version developed in [36] which requires extra one iteration.

For the second large transmission network TCase9241, the variant NR-c-car [36] diverges whereas other versions converge. Furthermore, NR-c-pol and NR-c-car versions developed in this paper converge after only three iterations whereas other versions (NR-p-pol [2], NR-p-car and NR-p-car [33]) need five to six iterations. For the largest transmission network TCase13659, both versions NR-p-car [33] and NR-c-car [36] diverge whereas all variants (NR-p-car, NR-c-pol and NR-c-car) developed in this paper and NR-p-pol [2] find the solution. Additionally, these four converged versions 


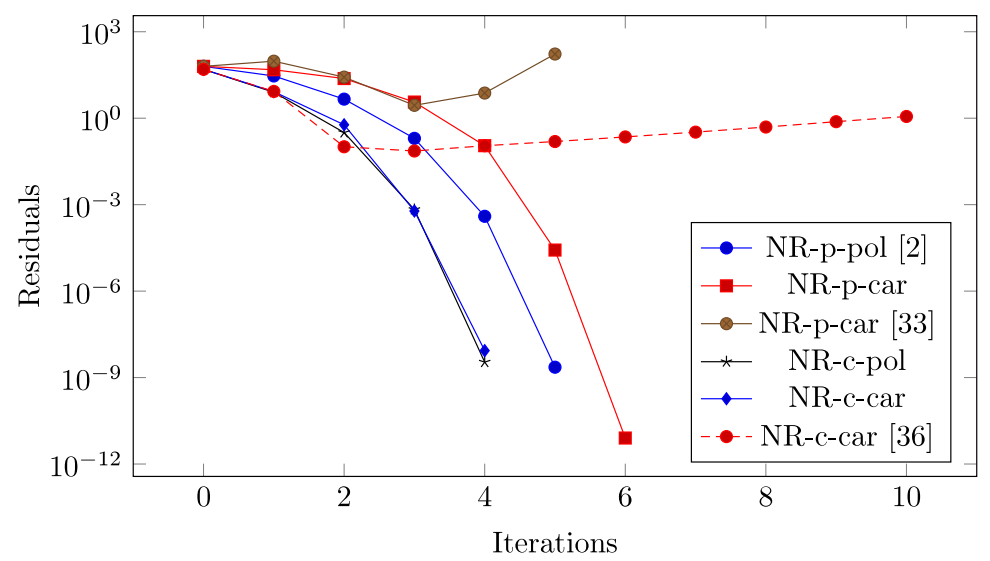

Fig. 1. Convergence of all Newton power flow versions on transmission network TCase13659.

have a quadratic convergence as shown in Fig. 1. Moreover, NR-c-pol and NR-c-car variants converge faster than the most famous variant NR-p-pol [2] in terms of iterations for both large transmission networks. Thus, we can conclude that polar current-mismatch (NR-c-pol) and Cartesian current-mismatch (NR-c-car) variants developed in this paper are more preferable for large transmission power flow problems.

\section{Conclusion}

In this paper, we formulate and analyze the Newton based power flow methods that are used for the power flow computation on balanced distribution and transmission networks. For the various methods we consider two different mismatch functions: the current and power balance equations and three different coordinate systems: Cartesian, polar and complex form. This leads to six different versions of the Newton power flow method. Studying these versions in a common framework enables us to analyze and compare all variants in a unified way. Furthermore, the existing variants of the Newton power flow method developed in $[2,33,36]$ are implemented and compared with the newly developed/improved versions of the Newton power flow method (Cartesian power-mismatch, polar current-mismatch, Cartesian currentmismatch and complex current-mismatch). In case of the polar and Cartesian current-mismatch versions, the reactive power $Q$ is chosen as a dependent variable for each PV bus. Thus, we compute the correction $\Delta Q$ at each iteration and update $Q$ using the computed corrections. Eqs. (2) and (3) are used instead of the voltage-magnitude-squared mismatch equation (1) in versions using Cartesian coordinates. The order of the Jacobian matrix equation is $\left(2 N-N_{g}-2\right)$ for the versions using the power-mismatch function whereas versions using the current-mismatch function have $(2 \mathrm{~N}-2)$ linear equations.

The polar current-mismatch and Cartesian current-mismatch variants of the Newton power flow method that are developed in this paper deliver the best result for both distribution and transmission networks. Therefore, we encourage power system operators to apply these two variants for power flow computations on balanced distribution and transmission networks.

In addition, the Cartesian current-mismatch version has an advantage in the calculation of the Jacobian matrix because its off-diagonal elements are constant and equal to the terms of the nodal admittance matrix. Moreover, depending on the properties of the given network, one version can work better than others. Therefore, it is crucial to study which version is more suitable for what kind of power networks. In the near future, these newly developed versions will be applied to three-phase power flow problems on unbalanced distribution networks and will be implemented in Matpower.

\section{Acknowledgment}

This research is supported by NWO (the Netherlands Organization for Scientific Research), domain Applied and Engineering Sciences, Grant No 14181.

\section{Appendix. Notation}

$N$ : number of buses in the network

$N_{g}$ : number of generator buses

$h$ : iteration counter

$V_{k}=V_{k}^{r}+\imath V_{k}^{m}$ : complex voltage at bus $k$ 


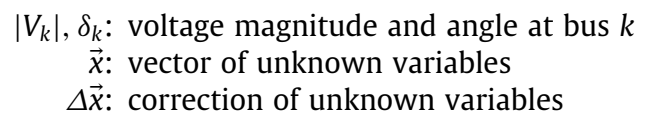

$F_{k}(\vec{x})$ : complex power or current mismatch function at bus $k$

$J(\vec{x})$ : Jacobian matrix of the mismatch function

$\Delta S_{k}=\Delta P_{k}+\imath \Delta Q_{k}$ : complex power mismatch at bus $k$

$\Delta I_{k}=\Delta I_{k}^{r}+\imath \Delta I_{k}^{m}:$ complex current mismatch at bus $k$

$S_{k}^{s p}=P_{k}^{s p}+\imath Q_{k}^{s p}$ : specified complex power at bus $k$

$S_{k}^{G}=P_{k}^{G}+\imath Q_{k}^{G}$ : generated complex power at bus $k$

$S_{k}^{L}=P_{k}^{L}+\imath Q_{k}^{L}:$ complex power load at bus $k$

$Y_{i k}=G_{i k}+\imath B_{i k}:(i, k)$ the element of nodal admittance matrix

\section{References}

[1] H.W. Hale, J.B. Ward, Digital computer solution of power flow problems, AIEE Trans. pt. III Power App. Syst. 75 (1956) $398-402$.

[2] William F. Tinney, Clifford E. Hart, Power flow solution by newton's method, IEEE Trans. Power App. Syst. (11) (1967) 1449-1460.

[3] Homer E. Brown, Gordon K. Carter, Harvey H. Happ, Conrad E. Person, Z-matrix algorithms in load-flow programs, IEEE Trans. Power Appar. Syst. (3) (1968) 807-814.

[4] Brian Stott, Of Alsaç, Fast decoupled load flow, IEEE Trans. Power App. Syst. (3) (1974) 859-869.

[5] Robert A.M. Van Amerongen, A general-purpose version of the fast decoupled load flow, IEEE Trans. Power Syst. 4 (2) (1989) 760-770.

[6] A.J. Monticelli, Ariovaldo Garcia, O.R. Saavedra, Fast decoupled load flow: hypothesis, derivations, and testing, IEEE Trans. Power Syst. 5 (4) (1990) 1425-1431.

[7] Hasan Dag, Adam Semlyen, A new preconditioned conjugate gradient power flow, IEEE Trans. Power Syst. 18 (4) (2003) $1248-1255$.

[8] Fan Zhang, C.S. Cheng, A modified newton method for radial distribution system power flow analysis, IEEE Trans. Power Syst. 12 (1) (1997) 389-397.

[9] Alexander J. Flueck, Hsiao-Dong Chiang, Solving the nonlinear power flow equations with an inexact newton method using gmres, IEEE Trans. Power Syst. 13 (2) (1998) 267-273.

[10] P.A.N. Garcia, J.L.R. Pereira, S. Carneiro, V.M. da Costa, N. Martins, Three-phase power flow calculations using the current injection method, IEEE Trans. Power Syst. 15 (2) (2000) 508-514.

[11] V.M. Da Costa, J.L.R. Pereira, N. Martins, An augmented newton-raphson power flow formulation based on current injections, Int. J. Elec. Power Energy Syst. 23 (4) (2001) 305-312.

[12] F. De Leon, A. Semlyen, Iterative solvers in the newton power flow problem: preconditioners, inexact solutions, and partial jacobian updates, IEE Proc., Gener. Transm. Distrib. 149 (4) (2002) 479-484.

[13] U Thongkrajay, N Poolsawat, T Ratniyomchai, T Kulworawanichpong, Alternative newton-raphson power flow calculation in unbalanced threephase power distribution systems, in: Proceedings of the 5th WSEAS international conference on Applications of electrical engineering, World Scientific and Engineering Academy and Society (WSEAS), 2006, pp. 24-29.

[14] Lei Wang, Chen Chen, Tao Shen, Improvement of power flow calculation with optimization factor based on current injection method, Discrete Dyn. Nat. Soc. 2014 (2014).

[15] R. Idema, D. Lahaye, Computational Methods in Power System Analysis, Atlantis Studies in Scientific Computing in Electromagnetics, Atlantis Press, 2014, https://books.google.nl/books?id=0Df0sgEACAAJ.

[16] Brian Stott, Review of load-flow calculation methods, Proc. IEEE 62 (7) (1974) 916-929.

[17] Dariush Shirmohammadi, H.W. Hong, A. Semlyen, G.X. Luo, A compensation-based power flow method for weakly meshed distribution and transmission networks, IEEE Trans. Power Syst. 3 (2) (1988) 753-762.

[18] G.X. Luo, A. Semlyen, Efficient load flow for large weakly meshed networks, IEEE Trans. Power Syst. 5 (4) (1990) $1309-1316$.

[19] T.-H. Chen, M.-S. Chen, Toshio Inoue, Paul Kotas, Elie A Chebli, Three-phase cogenerator and transformer models for distribution system analysis, IEEE Trans. Power Deliv. 6 (4) (1991) 1671-1681.

[20] M.H. Haque, Efficient load flow method for distribution systems with radial or mesh configuration, IEE Proc., Gener. Transm. Distrib. 143 (1) (1996) 33-38.

[21] Cao Longjian Samuel Mok, Mma Salama, A new approach for power flow analysis of balanced radial distribution systems, Electr Mach. Power Syst. 28 (4) (2000) 325-340, http://dx.doi.org/10.1080/073135600268298.

[22] Jianwei Liu, M.M.A. Salama, R.R. Mansour, An efficient power flow algorithm for distribution systems with polynomial load, Int. J. Electr. Eng. Educ. 39 (4) (2002) 371-386.

[23] Jen-Hao Teng, A direct approach for distribution system load flow solutions, IEEE Trans. Power Deliv. 18 (3) (2003) 882-887.

[24] Ulas Eminoglu, M.H.akan Hocaoglu, A new power flow method for radial distribution systems including voltage dependent load models, Electr. Power Syst. Res. 76 (1) (2005) 106-114.

[25] S. Satyanarayana, T. Ramana, S. Sivanagaraju, G.K. Rao, An efficient load flow solution for radial distribution network including voltage dependent load models, Electr. Pow. Compo. Syst. 35 (5) (2007) 539-551.

[26] J.-H. Teng, Modelling distributed generations in three-phase distribution load flow, IET Gener. Transm. Dis. 2 (3) (2008) 330-340.

[27] Tsai-Hsiang Chen, Nien-Che Yang, Loop frame of reference based three-phase power flow for unbalanced radial distribution systems, Electr. Pow. Syst. Res. 80 (7) (2010) 799-806.

[28] Hongbo Sun, Daniel Nikovski, Tetsufumi Ohno, Tomihiro Takano, Yasuhiro Kojima, A fast and robust load flow method for distribution systems with distributed generations, Energy Procedia 12 (2011) 236-244.

[29] M.S. Srinivas, Distribution load flows: a brief review, in: Power Engineering Society Winter Meeting, 2000. IEEE, vol. 2, 2000, pp. 942-945 vol.2.

[30] Juan A. Martinez, Jean Mahseredjian, Load flow calculations in distribution systems with distributed resources. a review, in: Power and Energy Society General Meeting, IEEE, 2011, pp. 1-8.

[31] K. Balamurugan, Dipti Srinivasan, Review of power flow studies on distribution network with distributed generation, in: Power Electronics and Drive Systems (PEDS), IEEE Ninth International Conference on, IEEE, 2011, pp. 411-417.

[32] Ulas Eminoglu, M.H.akan Hocaoglu, Distribution systems forward/backward sweep-based power flow algorithms: a review and comparison study, Electr. Pow. Compo. Sys. 37 (1) (2008) 91-110.

[33] James E. Van Ness, John H. Griffin, Elimination methods for load-flow studies, Trans. Am. Inst. Electr. Eng. Part 3 80 (3) (1961) 299-302.

[34] Hieu Le Nguyen, Newton-raphson method in complex form, IEEE Trans. Power Syst. 12 (3) (1997) 1355-1359. 
[35] H.W. Dommel, W.F. Tinney, W.L. Powell, Further developments in Newton's method for power system applications, in: IEEE Winter Power Meeting, Conference Paper, CP 161-PWR New York, 1970.

[36] V.M. da Costa, N. Martins, J.L.R. Pereira, Developments in the newton raphson power flow formulation based on current injections, IEEE Trans. Power Syst. 14 (4) (1999) 1320-1326.

[37] P. Schavemaker, L. van der Sluis, Electrical Power System Essentials, Wiley, 2008, https://books.google.nl/books?id=-MnG05siaG0C.

[38] Mesut E. Baran, Felix F. Wu, Network reconfiguration in distribution systems for loss reduction and load balancing, IEEE Trans. Power Deliv. 4 (2) (1989) 1401-1407.

[39] Mesut E. Baran, Felix F. Wu, Optimal capacitor placement on radial distribution systems, IEEE Trans. Power Del. 4 (1) (1989) $725-734$.

[40] C.E. Murillo-Sanchez R. D. Zimmerman, R.J. Thomas, Matpower: steady-state operations, planning and analysis tools for power systems research and education, IEEE Trans. Power Syst. 26 (2011) 12-244. 